\title{
Bilateral Artificial Iris Implantation In The Patient With Congenital Aniridia, Cataract And Nystagmus. A Case Report.
}

\author{
Adam Cywinski and Katarzyna Lewicka
}

\begin{abstract}
The article describes a case of surgical treatment of a patient with congenital aniridia, progressive cataract and nystagmus. The patient underwent several stages of treatment, concluded with an artificial iris implantation in both eyes. The presence of significant nystagmus caused the appearance of postoperative complications in one eye, which is why changes in the method of further treatment were introduced.
\end{abstract}

Key words-congenital aniridia, artificial iris, cataract, nystagmus, myopia.

\section{INTRODUCTION}

Aniridia is a partial or complete absence of the iris. It can be congenital or acquired. The congenital form affects both eyes and, in $2 / 3$ of cases it is inherited in a dominant mode of inheritance. It may accompany organ disorders. Depending on the type of mutation, aniridia may be accompanied by a kidney tumor (WAGR syndrome) or cerebellar ataxia (Gillespie syndrome). The exact cause of aniridia is unknown, but its occurrence is associated with a mutation within the PAX6 gene (location 11p13). The participation of other genes, e.g. TRIM44, ITPR1, is also studied in the pathogenesis of aniridia. Research shows that the average incidence of aniridia is 1.8 per 100,000 births, equally affecting women and men [1]. Total or subtotal congenital aniridia causes photophobia and low visual acuity. These symptoms have been observed since early childhood, similarly to strabismus and nystagmus. Aniridia may be accompanied by eyelid ptosis, microcornea, corneal degeneration associated with aniridia (AAK), disorders of the Meibomian glands, glaucoma, persistent pupillary membrane, lens subluxation, cataract, hypoplasia of the optic disc and hypoplasia of the fovea. Gonioscopy performed in the case of congenital aniridia may reveal the presence of anomalies in the iridocorneal angle: Schlemm's canal dysgenesis and trabecular meshwork [1]. Nystagmus is one of the symptoms associated with aniridia. Its cause is probably in the abnormally developed optic disc or macular hypoplasia. Therefore, it is difficult to predict the extent of improvement in postoperative visual acuity. In their study E. F. Lopez et al. [2] described the case of a 15-year-old girl, in whom, in the eye examination, in addition to the partial aniridia and cataract, significant nystagmus was also observed. Horizontal and rotational movements were partly reduced by convergence. The patient had cataract removal with artificial lens implantation, and then through the same incision an artificial iris implant was inserted, which was placed in the ciliary sulcus. Each eye was treated according to the above procedure, at different times. Postoperative distance visual acuity has not improved. The binocular visual acuity of near vision significantly improved, examined using Snellen charts, postoperatively reaching 20/25 compared to 20/40 obtained before the procedure. The authors speculate that the effect of improved vision may be associated with blocking nystagmus by a convergence reflex.

Choosing an artificial iris implant in the eyes with congenital aniridia, a proper assessment of the local condition is a later indicator of the effectiveness of the procedure. With 3 variants of the location of the artificial iris in the eye, i.e. implantation into the sulcus, insertion of the lens into capsular bag or transscleral fixation, the choice should be dictated by the anatomical state of the interior and changes in eye motility. Surgeons do not always choose the option of artificial iris implantation. Jin da Wang et al [7] in 10 patients (17 eyes) with congenital aniridia, cataract and nystagmus, performed only cataract surgery with subsequent implantation of the capsule tension ring and intraocular lens into capsular bag. Instead of using an artificial iris, the authors decided to use prosthetic contact lenses with an iris drawing. They obtained subjective improvement of vision, reduction of photophobia and improvement of visual acuity from $1.0+/-0.2 \log$ MAR to $0.78+/-0.26 \log$ MAR. During 24 months of follow-up, patients tolerated contact lenses well. There were no complications such as glaucoma, corneal decompensation, endothelial cell loss or implant displacement, described by other authors in their publications [2]-[6],[8],[9].

\section{MATERIAL AND METHODS}

In November 2017, a 43-year-old female patient came to the clinic of the Silesian Eye Treatment Center in Zory (Poland) due to the progressive deterioration of visual acuity in both eyes. The consultation was aimed at answering the question whether there is a chance to improve her vision. The patient was born with congenital aniridia. Practically from birth, she struggled with poor vision and ubiquitous photophobia. Accompanying nystagmus and progressive myopia intensified the decrease in visual acuity, especially distance vision. Over time, cataract appeared that resulted in further visual impairment. Despite progressive lens opacification, the doctors decided not to remove the lens due to significant damage to the eye structures. Visual acuity of both eyes was so poor that the patient was not able to read 
the information about the bus departure time at the bus stop, even from a close distance, despite many attempts to correct the existing defect, including myopia.

Physical examination. The best visual acuity (BCVA) for distance vision reached the following values: for the right eye: 3 letters from 1.5 meters, left eye: 5 letters from 2 meters, tested on logMAR charts. Visual acuity for near vision in both eyes, without correction reached the value of D-1.25, tested on Snellen charts. Intraocular pressure reached $13 \mathrm{mmHg}$ in both eyes.

Anterior segment. The pathology revealed the presence of slight clouding and peripheral vascularization of the cornea. Fragments of the normal structure of the iris were visible near the base of the iris, mainly in the temporal part (Fig. 1a,b).

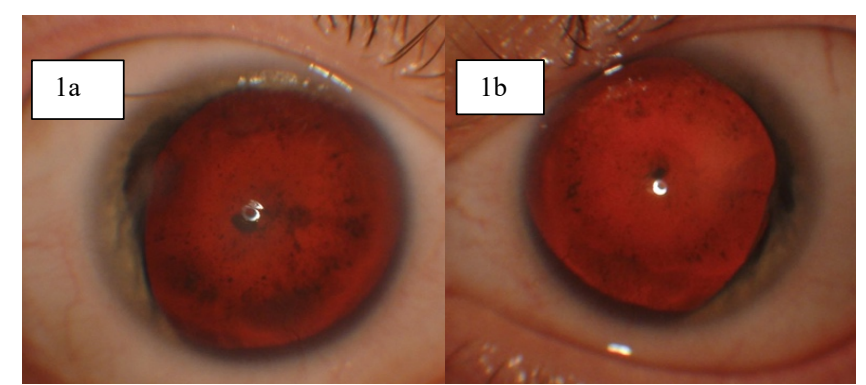

Fig.1 Congenital aniridia with cataract and myopia. Residual peripheral iris visible. a) right eye, b) left eye

Incomplete lens opacification allowed for proper examination of the posterior segment of the eye, although accompanying nystagmus significantly hindered it. Eye fundus examination did not reveal any retinal or vitreous pathologies. Axial length values for the right eye are $24.91 \mathrm{~mm}$, for the left eye $-25.72 \mathrm{~mm}$. The intraocular lens value was calculated using optical biometry, which was confirmed using immersion biometry.

After analyzing the local condition, the patient was qualified for surgical treatment of both eyes.

\section{RESULTS}

Starting from the right eye with poorer vision, a combined procedure, including cataract removal and implantation of both a monofocal lens and two partial iris implants into the capsule was proposed. (Fig. 2a,b).

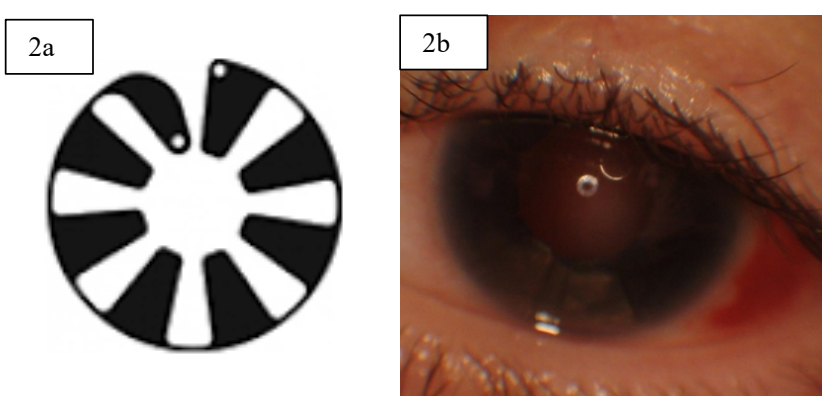

Fig. 2a) Partial iris implant by Morcher, b) Right eye. After insertion of two partial implants into the ciliary sulcus.

The procedure in the right eye was performed with one modification. Despite the standard diameter of capsulorhexis, the iris implants were not inserted into the capsule, so they were placed in the ciliary sulcus. This change has two major consequences. A larger than planned pupil diameter was obtained. Secondly, due to significant nystagmus, the iris implants were partially dislocated to the anterior chamber and they came into direct contact with corneal endothelium (Fig. 3).

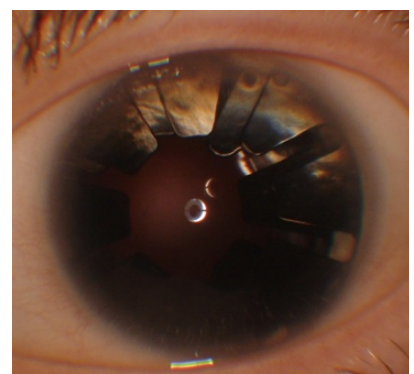

Figure 3. A month after surgery. Displacement of partial iris implants into the anterior chamber.

Because this condition could later lead to progressive corneal opacification, the doctors decided to modify the treatment. The patient was referred for a consultation to reduce nystagmus. The procedure was performed, and nystagmus was reduced. At that time, an artificial noncustomized iris was ordered for the patient, and bearing in mind residual nystagmus, model from Reper Company was chosen, which is suitable for transscleral fixation (Fig.4).

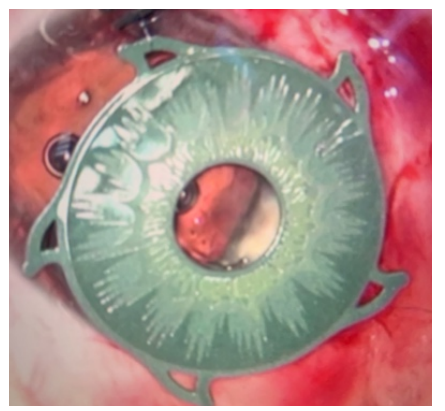

Fig. 4. Non-customized artificial iris by Reper. Due to the presence of a loops, this model is subject to transscleral fixation

The color of the iris was chosen by the patient.

The procedure was performed four months after surgical reduction of nystagmus. Partial iris implants were replaced with artificial iris implants, which were inserted directly behind the residual iris and fixated in three places to the sclera. In the absence of complications, three months later the same type of surgery was performed in the other eye. It involved combined cataract removal and implantation of a monofocal lens into the capsule with transscleral fixation of artificial iris (Fig. 5a,b). 


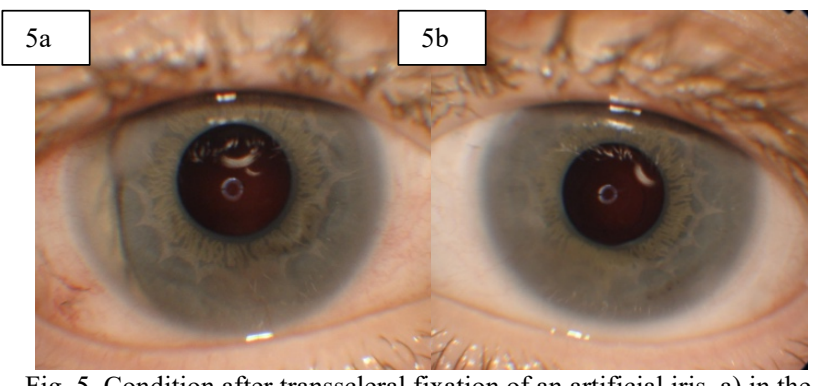

Fig. 5. Condition after transscleral fixation of an artificial iris, a) in the right eye, b) in the left eye

The video of both procedures can be watched on the following website: https://medtube.pl/okulistyka/filmymedyczne/24673 wszczepienie-sztucznych-teczowek-wobu-oczach.

Follow-up examinations carried out after 1, 3, 6 months and after a year showed gradual improvement in visual acuity and no visible complications associated with the performed procedures. Intraocular pressure did not change in subsequent follow-ups, reaching values in the range of 17-20 $\mathrm{mmHg}$ in both eyes. BCVA examined 10 months after the last procedure showed the following values: distance vision - 0.9 LogMAR in both eyes, near vision, using Snellen charts - D-1.0. The subjective quality of vision improved significantly, and photophobia subsided. Among the adverse effects that the patient reported immediately after the procedure, darker vision resulting from a decrease in the supply of light to the interior of the eye by introducing the iris effect, was observed.

\section{Discussion}

Analyzing this particular case and individual stages of treatment, several conclusions and doubts arise.

\section{A. Nystagmus}

To avoid subsequent adverse effects, it should be reduced in the first stage of treatment. Reducing nystagmus improves vision [12]. Another benefit is the reduction of the risk of subluxation and even dislocation of implants inserted into the lens capsule. When deciding to simultaneously insert an artificial lens and two partial iris implants into the lens capsule, one should not forget about their significant weight.

\section{B. Introduction of iris implants into the ciliary sulcus without additional fixation.}

This option increases the risk of implants displacement into the anterior chamber and subsequent irritation of the internal structures of the eye by micro-movements of the eyeball caused by nystagmus. As a complication, the loss of endothelial cells is listed, which can lead to edema and corneal decompensation [2,3]. In addition, the black color of iris implants is not very beneficial from an aesthetic point of view due to the "black iris" effect obtained.

C. The introduction of a complex implant correcting aniridia and postoperative aphakia at the same time, with the possibility of its fixation to the eye structures or insertion into capsular bag.
These are also options that should not be the treatment of choice in this particular case. The introduction of an intraocular lens into the capsule is the most physiological option. By introducing a complex implant into the capsule, which is heavier than the intraocular lens itself, we increase the risk of subsequent complications, including mainly subluxation of the lens. When applying fixation of a complex implant and its location in the ciliary sulcus, in case of its intolerance and the need to remove it, the eye is again aphakic and requires the secondary implantation of an artificial lens.

\section{CONCLUSION}

Considering the patient with nystagmus, congenital aniridia, in whom we additionally observe the presence of a nonsubluxated, cataract requiring removal, the proposal of the best treatment option involves two stages. The first stage involves the surgical reduction of nystagmus, which allows better vision to be obtained, and above all prevents subsequent postoperative complications, mainly subluxation of implants. The next stage involves the removal of cataract and the insertion of an artificial lens into the lens capsule, combined with the transscleral fixation of an artificial noncustomized iris. Bilateral congenital aniridia does not require the use of more expensive customized products, because we do not have to choose the color of the iris of the eye operated on to suit the healthy eye. A cheaper alternative to implantation of an artificial iris is the introduction of a black aniridia iris implant, a model that can be fixated. However, a disadvantage is the huge scleral incision (about $10 \mathrm{~mm}$ ) that needs to be made to insert the implant into the eye.

\section{REFERENCES}

[1] Tripathy K, Salini B „Aniridia”; Review from Stat Pearls Publishing, Treasure Island (FL), 09 Mar 2019; PMID: 30844160

[2] Esther Fernandez-Lopez, Francisco Pastor Pascual, Marta PerezLopez, Alejandro Madrigal Quevedo, and Cristina Peris Martınez" Sutureless Artificial Iris after Phacoemulsification in Congenital Aniridia"; Optometry and Vision Science, Vol. 92, No. 4S, April 2015

[3] Martin Stephan Spitzer,1* Anja Nessmann,1* Julia Wagner,1 Efdal Yoeruek,1 Karl Ulrich Bartz-Schmidt,1 Peter Szurman2 and Gesine B. Szurman2 "Customized humanoptics silicone iris prosthesis in eyes with posttraumatic iris loss: outcomes and complications"; Acta Ophthalmol. 2016: 94: 301-306

[4] Xiaodi Qiu, Yinghong Ji, Tianyu Zheng and Yi Lu "The efficacy and complications of black diaphragm intra-ocular lens implantation in patients with congenital aniridia"; Acta Ophthalmol. 2016: 94: e340e344

[5] Vahid Jusufovic1, Emir Cabric2, Allen Popovic-Beganovic1, Zlatko Musanovic1, Jasmin Zvornicanin1 "Treatment of Congenital Aniridia Associated with Subluxated Infantile Cataract"; Med Arh. 2014 Jun; 68(3): 212-214

[6] Efdal Yoeruek1, Karl U. Bartz-Schmidt1: "A new knotless technique for combined transscleral fixation of artificial iris, posterior chamber intraocular lens, and penetrating keratoplasty"; Eye (2019) 33:358362

[7] Jin Da Wang1, Jing Shang Zhang1, Ying Xiong2, Jing Li2, Xiao Xia Li1, Xue Liu1, Jing Zhao1, Frank F. Tsai3, Jhanji Vishal4, Qi Sheng You1, Yao Huang2 and Xiu Hua Wan 1 *" Congenital aniridia with cataract: case series"; BMC Ophthalmology (2017) 17:115

[8] Yehia S. Mostafa1, Amr A. Osman1, Dina H. Hassanein1, Ashraf M. Zeid2, Ahmed M. Sherif1 "Iris reconstruction using artificial iris prosthesis for management of aniridia"; European Journal of Ophthalmology 2018, Vol. 28(1) $103-107$ 
[9] https://www.eyeworld.org/update-artificial-iris-implants

[10] http://ziemerusa.com/artificial-iris/

[11] Penix K, Swanson MW, DeCarlo DK. Nystagmus in pediatric patients: interventions and patient-focused perspectives. Clin Ophthalmol. 2015 Aug 21;9:1527-36. doi: 10.2147/OPTH.S62786. eCollection 2015. Review.

Adam Cywinski, date of birth 22.09.1967. Medical Director and founder of private medical center, Silesian Eye Treatment Centre, Zory, Poland. Consultant of posterior segment diseases. He graduated Silesian Medical Academy in Katowice and gained medical degree in the biggest Clinic of Ophthalmology, also in Katowice. He doctorated in surgical topic "Posterior vitrectomy with gas endotamponade and ILM peeling in IV stage macular hole, in 2003. As a surgeon he made a few thousands posterior vitrectomy procedures, including macular diseases, retinal detachment, diabetic and posttraumatic cases. His next interest is anterior segment surgery with special focus on reconstruction of the iris. His everyday job includes cataract surgery of complicated cases and surgery with premium class lenses. Dr Cywinski is a member of PTO, AAO, ESCRS, Euretina.

Katarzyna Lewicka - medical studies on Silesian Medical University. In 2007 PhD - "Corneal morphology assessment using confocal microscopy in people using contact lenses", Works as ophthalmologist in Silesian Eye Treatment Centre, Zory. Especially interest in examination of children with ophthalmic and neurological disorders. 